\title{
Selective differentiation of bone marrow-derived mesenchymal stromal cells into osteocytes via endochondral ossification in an apatite-fiber scaffold
}

\author{
Michiyo HONDA, ${ }^{, \dagger}$ Yuka WATANABE, ${ }^{* *}$ Takahide TSUCHIYA, ${ }^{* *}$ \\ Nobuyuki KANZAWA** and Mamoru AIZAWA ${ }^{* * * *}$ \\ *Aizawa "Next-generation Bioceramics" Project, Kanagawa Academy of Science and Technology (KAST), \\ 3-2-1 Sakado, Takatsu-ku, Kawasaki 213-0012, Japan \\ ${ }^{* *}$ Department of Materials and Life sciences, Faculty of Science and Technology, Sophia University, \\ 7-1 Kioi, Chiyoda-ku, Tokyo 102-8554, Japan \\ *** Department of Applied Chemistry, School of Science and Technology, Meiji University, \\ 1-1-1 Higashimita, Tama-ku, Kawasaki 214-8571, Japan
}

\begin{abstract}
Bone marrow-derived mesenchymal stromal cells (BMSCs) are pluripotent progenitor cells that can regenerate different skeletal tissues in response to environmental signals. Scaffolds play a critical role in tissue engineering, and their microstructure is essential for inductive bone formation. In the present study, we have used highly porous, structurally stable three-dimensional apatite-fiber scaffolds (AFSs) and investigated their ability to support cell proliferation and differentiation. BMSCs in/on AFSs were proliferated in a three-dimensional manner when both micro- and macro-pores were present. The characteristic structure of the AFS enhanced calcification, production of extracellular matrix (ECM), and osteogenic differentiation, especially endochondral ossification. Furthermore, BMSCs cultured in/on AFSs did not undergo adipogenesis and selectively underwent osteogenic differentiation. These results suggest that AFSs provide a suitable environment for bone regeneration and have potential applications for tissue engineering.
\end{abstract}

(C)2013 The Ceramic Society of Japan. All rights reserved.

Key-words : Scaffold, Hydroxyapatite, Bone marrow-derived stromal cell, Endochondral ossification, Differentiation

[Received May 15, 2013; Accepted June 3, 2013]

\section{Introduction}

Bone tissue engineering is a therapeutic approach that aims to regenerate or repair bone tissue using a scaffold, cells, and inductive factors to actively stimulate tissue formation. ${ }^{1)-4)}$ Three-dimensional scaffolds in particular provide the necessary support for cells to proliferate and maintain their capacity to differentiate. Scaffolds containing bone marrow-derived osteoprogenitors can be incorporated into implants to enhance bone repair. ${ }^{5), 6)}$ The scaffold is intended to mimic the native in vivo microenvironment, and this demands construction of bioactive scaffolds that are also capable of supporting vascularization, cell proliferation, and osteogenic differentiation. ${ }^{7}$ Matrices with three-dimensional porous structure have already been produced by various processes and have been constructed with different porosity, pore size, pore-size distribution, pore type (closed or open pore), and pore interconnectivities. These parameters play an important role in their biocompatibility.

Hydroxyapatite $\left[\mathrm{Ca}_{10}\left(\mathrm{PO}_{4}\right)_{6}(\mathrm{OH})_{2} ; \mathrm{HAp}\right]$, a bioactive ceramic, is a widely used biomaterial. Previously, we synthesized singlecrystal HAp fibers with long-axis dimensions of 100-200 $\mu \mathrm{m}$ using a homogenous precipitation method. ${ }^{8), 9)}$ Using these HAp fibers, we succeeded in fabricating an apatite-fiber scaffold (AFS) with well-controlled pore sizes of approximately $250 \mu \mathrm{m}$ (porosity of $98-99 \%) .{ }^{10)}$ Additionally, our recent study showed that AFSs

$\dagger$ Corresponding author: M. Honda; E-mail: ap-honda@newkast. or.jp can provide a three-dimensional culture environment and facilitate cell proliferation and differentiation using two different pores. ${ }^{11)}$

In recent years, mesenchymal stem cells (MSCs) have attracted much interest due to their self-renewing potential and multipotentiality for clinical use. ${ }^{12-14)}$ Bone marrow-derived MSCs (BMSCs) grow as foci with a fibroblast-like morphology and are termed colony-forming unit fibroblasts. BMSCs are currently among the best characterized adult stem cells and can be isolated from various tissue sources such as fat, muscle, and bone. These cells are able to differentiate into bone, fat, cartilage, muscle tissue, and neurons. ${ }^{15)}$

Bone development occurs via two distinct cellular and molecular pathways: intramembranous and endochondral ossification. In intramembranous ossification, BMSCs condense and directly differentiate into bone-forming osteoblasts, in contrast to endochondral ossification in which BMSCs condense and become chondrocytes. Cartilage anlagen directs the formation of osteoblasts that ultimately form woven bone in the developing axial and appendicular skeleton. ${ }^{16)}$ The majority of in vivo bone formation occurs via endochondral ossification. ${ }^{17}$ Endochondral ossification begins as BMSCs differentiate into chondrocytes and form a matrix template in the region of the prospective bone. Then, as chondrocytes within this template progress through a spatially and temporally specific series of phases including proliferation, prehypertrophy, hypertrophy, and finally apoptosis, additional BMSCs infiltrate the growth plate and begin to differentiate toward osteogenic lineages, replacing the cartilage template with ossified bone. ${ }^{18)}$ 
In the present study, we have developed three-dimensional AFSs and analyzed the mechanism of ossification using BMSCs.

\section{Experimental}

\subsection{Fabrication and characterization of AFSs}

A fibrous HAp was prepared as previously reported. ${ }^{19)}$ HAp fibers were suspended with spherical carbon beads (Nika beads; Nihon Carbon Company) with a diameter of $\sim 150 \mu \mathrm{m}$ in the mixed solvent [ethanol/water $=1 / 1(\mathrm{v} / \mathrm{v})$ ] at a $20 / 1$ carbon/ HAp (w/w) ratio. After stirring and blending the slurry homogeneously, green compacts for the scaffold were fabricated by pouring and vacuum pumping the slurry into a metallic mold ( 7 or $15 \mathrm{~mm}$ internal diameter). Compacts were fired at $1300^{\circ} \mathrm{C}$ for $5 \mathrm{~h}$ in a stream atmosphere. AFS was shaped $7 \mathrm{~mm}$ or $15 \mathrm{~mm}$ in diameter and $5 \mathrm{~mm}$ in height. AFS $(7 \mathrm{~mm})$ was used for morphological observation and another was used for evaluation of gene expression.

Scaffolds were characterized with X-ray diffractometry (XRD; MiniFlex, Rigaku, Japan) and scanning electron microscopy (SEM; JSM-6390LA, JEOL, Japan). XRD patterns were obtained using $\mathrm{Cu} \mathrm{K} \alpha$ radiation with a $\theta / 2 \theta$ diffractometer. Data were collected in the range of $2 \theta=4-50^{\circ}$ with a step size of $4^{\circ} / \mathrm{min}$. The crystalline phase was identified using the JCPDS reference data. The morphology of the samples was observed with SEM at an accelerating voltage of $15 \mathrm{kV}$.

\subsection{Culture of BMSCs}

BMSCs were prepared from the femur and tibia of 4-week-old Wistar rats using the method reported by Maniatopoulos et al. ${ }^{20)}$ Cells were seeded into tissue culture dishes (BD, Franklin Lake, $\mathrm{NJ}$ ) and cultured in $\alpha$-MEM (minimal essential medium, Sigma, St. Louis, MO) containing 10\% fetal bovine serum (FBS, Sigma) and $0.1 \%$ antibiotics (penicillin-streptomycin solution, Sigma) at $37^{\circ} \mathrm{C}$ in $5 \% \mathrm{CO}_{2}$. The culture medium was changed once every 2-3 days until the cells were almost confluent. The cells were trypsinized with trypsin-EDTA (Sigma) to produce a cell suspension, which was seeded by adding it dropwise on top of the AFS that was placed at the bottom of the wells in a 6-well plate (Nalge Nunc International, Rochester, NY). The culture medium in the wells was refreshed every two days, and the culture was maintained for a maximum of 28 days. Subconfluent secondpassage cells were used for all experiments. Cells were cultured in regular medium ( $\alpha$-MEM supplemented with $10 \%$ FBS) or in differentiation medium (regular medium plus $20 \mathrm{mM} \beta$-glycerol phosphate, $4 \mathrm{mM}$ L-ascorbic acid, $10 \mathrm{nM}$ dexamethasone) for appropriate periods.

\subsection{Preparation of frozen sections of cells cultured on AFSs}

BMSCs cultured on AFSs were fixed in $4 \%$ paraformaldehyde for $15 \mathrm{~min}$. Samples were washed with phosphate-buffered saline (PBS) and immersed in $1 \%(\mathrm{w} / \mathrm{v})$ gelatin/PBS solution for 60 min under reduced pressure. The specimens were embedded in O.C.T. compound (Sakura Tissue-Tek), rapidly frozen in liquid nitrogen, and stored at $-20^{\circ} \mathrm{C}$. Sections $(18 \mu \mathrm{m})$ were prepared using a cryostat (HM505E, Carl Zeiss Co., Ltd., Oberkochen, Germany) and placed onto Superfrost/Plus microscope slides (Fisher Scientific, Pittsburgh, PA).

\subsection{Light and confocal laser scanning microscopy}

Sections prepared from cultured samples were stained with Alizarin Red, Alcian Blue, or Sudan IV and examined with a light microscope (Axiovert 135, Carl Zeiss Co., Ltd.).
Table 1. Primers used in this study

\begin{tabular}{ccl}
\hline Gene & & \multicolumn{1}{c}{ Sequence $\left(3^{\prime}-5^{\prime}\right)$} \\
\hline GAPDH & $\begin{array}{c}\text { forward } \\
\text { reverse }\end{array}$ & $\begin{array}{l}\text { ACCACAGTCCATGCCATCAC } \\
\text { TCCACCACCCTGTTGCTGTA }\end{array}$ \\
Col I & $\begin{array}{c}\text { forward } \\
\text { reverse }\end{array}$ & $\begin{array}{l}\text { GAACTTGGGGCAAGACAGTCA } \\
\text { GTCACGTTCAGTTGGTCAA }\end{array}$ \\
Col II & $\begin{array}{c}\text { forward } \\
\text { reverse }\end{array}$ & GGAAAGTCTGGGGAAAGAGG \\
& CAGTCCCTGGGTACCAGAA \\
Col X & forward & CAGTGCTAACCAGGGGGTAA \\
& reverse & ATGGGAGCCACTAGGAATCCTGA \\
OC & forward & ATGAGGACCCTCTCTCTGCTC \\
& reverse & GTGGTGCCATAGATGCGCTTG \\
Aggrecan & forward & TAGAGAAGAAGAGGGGTAGG \\
& reverse & AGCACTAGGAGCCAGGGTTAT \\
Runx 2 & forward & TGAGATTTGTGGGCCGGAGC \\
& reverse & GGGACACCTACTCTCATACTGG \\
Sox 9 & forward & AGGAAGCTGGCAGACCAGTA \\
& reverse & CCCTCTCGCTTCAGATCAAC \\
\hline
\end{tabular}

Sections for confocal laser scanning microscopy (CLSM) were further incubated in $0.1 \%$ TritonX-100/PBS for $10 \mathrm{~min}$ and washed with PBS. To visualize fibrous-actin (F-actin), we incubated samples for $30 \mathrm{~min}$ with Alexa Fluor 594-phalloidin (Life Technologies, Carlsbad, CA) diluted 1:200 in PBS. After washing with PBS, some sections were further stained with DAPI (Dojindo, Kumamoto, Japan) diluted 1:500 in PBS for 10 min to visualize nuclei. The rinsed samples were mounted with antifade mounting medium and examined with a CLSM (LSM Live 5, Carl Zeiss Co., Ltd.). All sections were prepared from top to middle of AFS.

\subsection{RNA preparation from cells grown on AFSs and cDNA synthesis}

AFSs with BMSCs were incubated for 7, 14, 21, or 28 days. After each incubation period, AFSs were washed with PBS, and total RNA was prepared from cells grown on AFSs or in a dish. Single-stranded cDNA was synthesized using a High Capacity cDNA Reverse Transcription kit (Applied Biosystems, Carlsbad, CA), following the manufacturer's instructions.

\subsection{Semi-quantitative reverse transcription-poly- merase chain reaction (RT-PCR)}

The expression of marker genes of osteogenic differentiation [i.e., Runx2, type I collagen ( $\mathrm{Col} \mathrm{I})$, and osteocalcin (OC)] and endochondral ossification [i.e., Sox9, aggrecan, type II collagen (Col II), and type X collagen $(\mathrm{Col} X)]$ was examined with semiquantitative RT-PCR. Glyceraldehyde 3-phosphate dehydrogenase $(G A P D H)$ gene expression was examined as an internal control. The obtained cDNA was amplified with primer sets described in Table 1.

\subsection{Statistical analysis}

All data were statistically analyzed with the Student's $t$ test and expressed as the mean \pm standard deviation (SD). $P<0.05$ was accepted as statistically significant.

\section{Results and discussion}

\subsection{Characterization of AFS}

Figure 1 shows SEM images [Figs. 1(a)-1(d)] and a macroscopic image [Fig. 1(e)]. AFSs showed two different types of pores: micro- and macro-pores [asterisks in Fig. 1(d)]. Micropores arose from random distribution of interspaces of the AFSs 

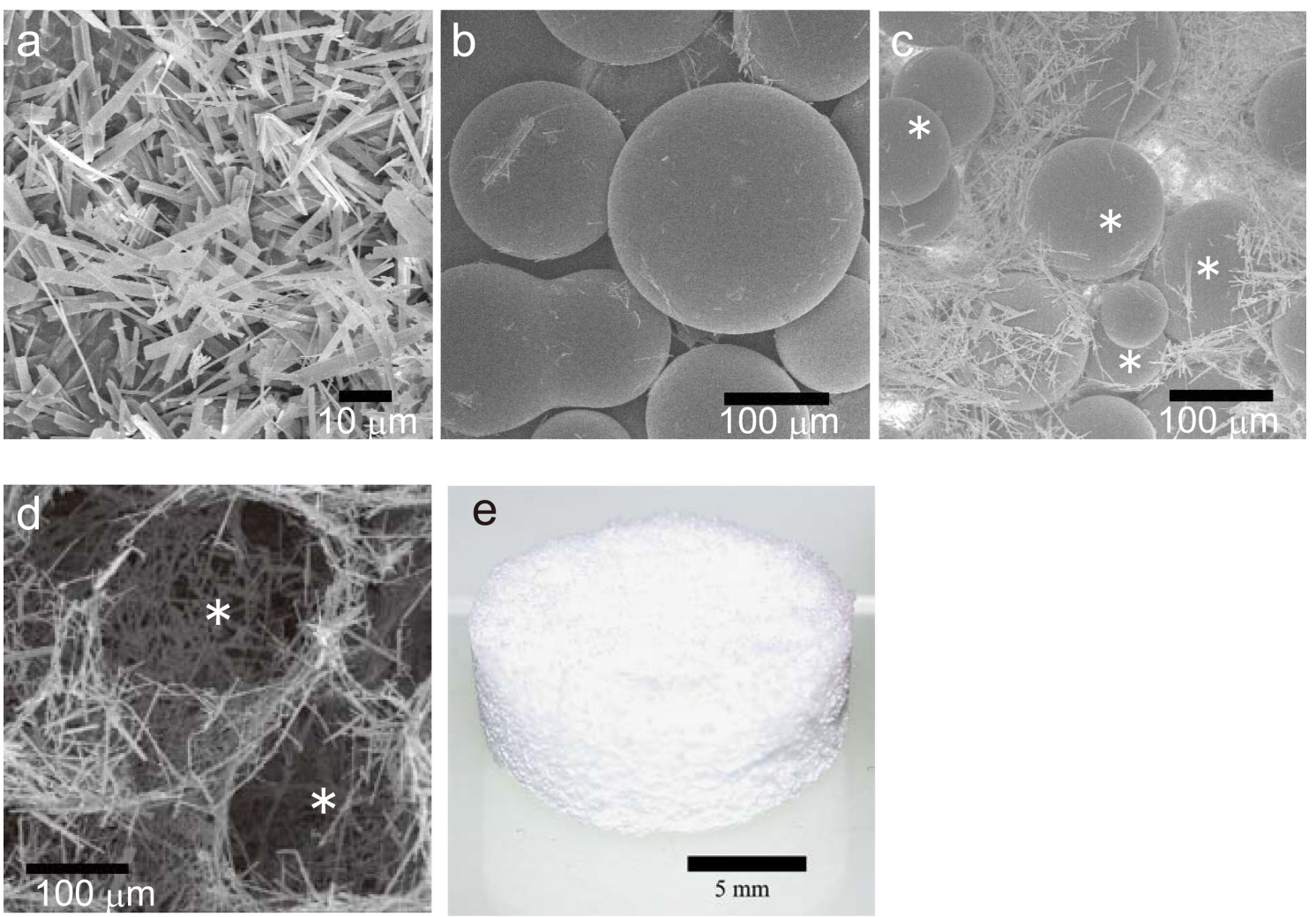

Fig. 1. Scanning electron micrographs of apatite fiber, carbon beads, or AFS and photograph of AFS. Carbon beads (b) were added to apatite fiber (a) and green compacts (c) were fired at $1300^{\circ} \mathrm{C}$ for $5 \mathrm{~h}$. The resulting AFS has two kinds of pores (microand macro pores). Macro pores were derived from carbon beads, indicating asterisks. The pore sizes of AFS are approximately $250 \mu \mathrm{m}$ and their porosity is $98-99 \%$. Bars indicate $10 \mu \mathrm{m}$ (a), $100 \mu \mathrm{m}(\mathrm{b}-\mathrm{d})$ and $5 \mathrm{~mm}(\mathrm{e})$.

and provided $10 \mu \mathrm{m}$ wide spaces for cells. The other larger pores ( $\sim 250 \mu \mathrm{m}$ width) were designated macro-pores and were formed after burning carbon beads out [asterisks in Fig. 1(c)], which were embedded during preparation of mixed compacts of AFS/ beads. These pores formed inter-pore connections, and the pore sizes were regarded as appropriate for trapping cells. Control of the chemical, topographical and mechanical properties of the AFS makes it possible to support cell attachment and to guide three-dimensional tissue formation [Fig. 1(e)].

Figure 2 illustrates the XRD pattern of the resulting AFS and clearly shows that the scaffold contained a single phase of HAp with high crystallinity (JCPDS 9-432).

\subsection{Distribution of BMSCs in AFSs during cell proliferation and differentiation}

Previous investigations demonstrated that preosteoblasts in a three-dimensional structure of the AFS are in a more natural environment than those in a normal, two-dimensional culture dish system. ${ }^{10)}$ To explore the formation of cellular networks in detail, we observed cell attachment and distribution in AFSs (Fig. 3). The cells were labeled by staining the actin cytoskeleton and nucleus, and serial images of BMSCs growing in AFSs were taken with a CLSM. After 14 days of culture in regular medium, BMSCs had adhered tightly on apatite fibers, and their microspikes had extended along the skeleton of the AFS [Figs. 3(a)-3(c)]. Furthermore, after 28 days, the micro-pores were filled with BMSCs, and cell-cell bridges had formed over the macro-pores [Figs. 3(d)-3(f)]. These results showed that the three-dimensional culture environment, which consists of microand macro-pores, could simulate the physiological condition in

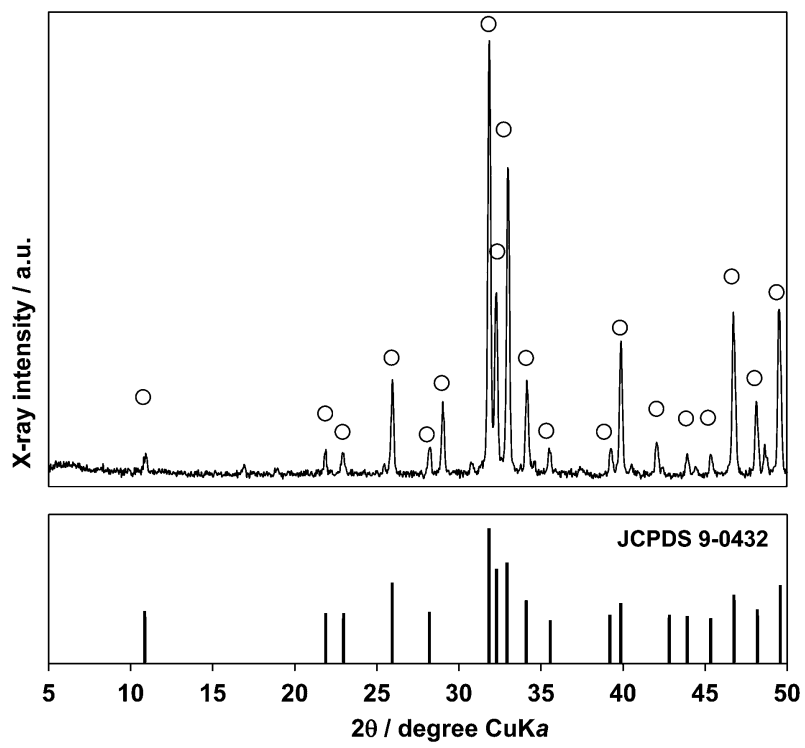

Fig. 2. Characterization of AFS. XRD patterns of AFS (upper panel). AFS was fired at $1300^{\circ} \mathrm{C}$ for $5 \mathrm{~h}$ in a stream atmosphere. The crystalline phases was identified as HAp (JCPDS, 9-0432, lower panel).

bone. In addition, optimal pore diameters on AFSs allowed cell migration and may permit optimal flow of body fluids.

\subsection{Observation of the extracellular matrix (ECM) and ossification of BMSCs in AFSs}

To observe calcification in AFSs, Alizarin Red staining for 

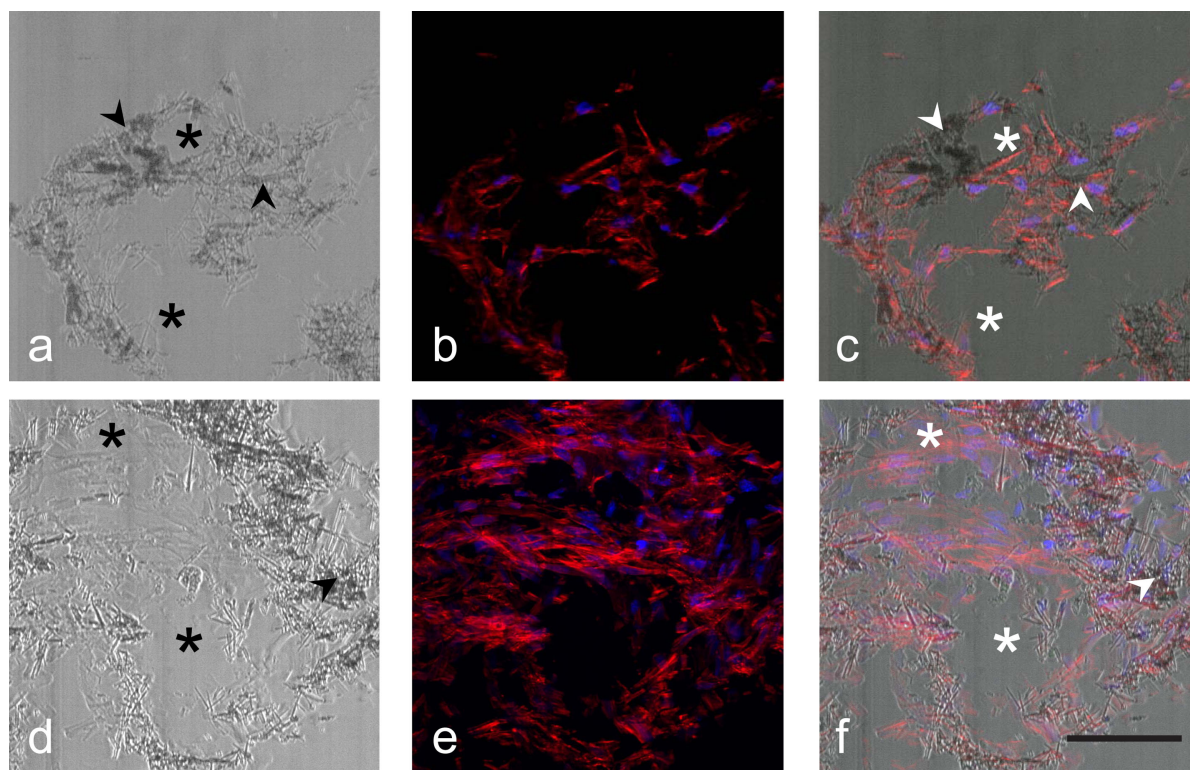

Fig. 3. Cell distribution on AFS during proliferation and differentiation. BMSCs were cultured for 14 or 28 days under regular condition. Scaffolds after culturing cells were fixed with $4 \%$ PFA, immersed in $1 \%$ gelatin, and embedded in OCT compound. Thin sections were reacted with Alexa594-pholloidin and DAPI (b, e) as described in Exprimental. Cells were fully extended and formed bridges within micro- (arrowheads) and macro-pores (asterisks). Bar indicates $100 \mu \mathrm{m}$.
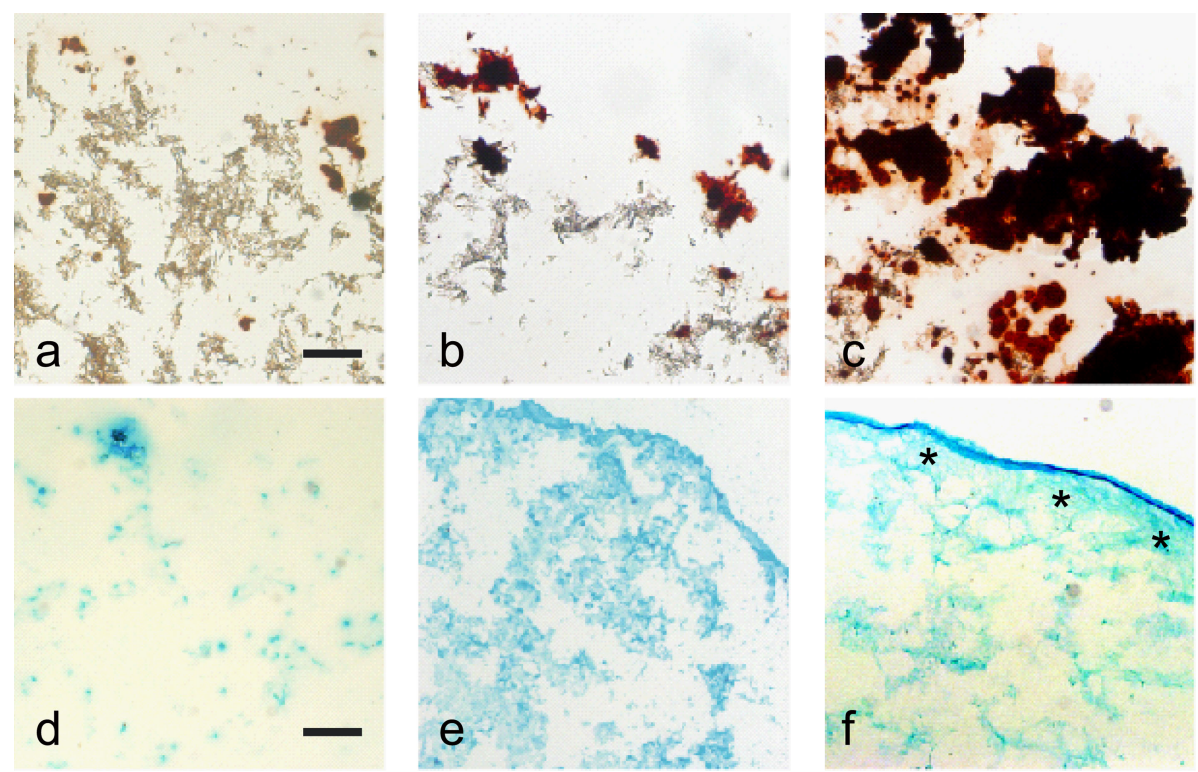

Fig. 4. Calcium deposition and ECM production of BMSCs-seeded AFS BMSCs were cultured for 7, 14, or 21 days in normal growth medium. Thin sections were reacted with Alizarin Red to show the calcification $(\mathrm{a}-\mathrm{c})$. Calcification was observed at periphery rather than center of AFS. Sections were also stained by Alcian Blue to visualize proteoglycan-rich ECM (d-f). A sulfated proteoglycan-rich ECM was detected in macro-pores at periphery of AFS (asterisks). Bar indicates $100 \mu \mathrm{m}$.

mineralization was carried out [Figs. 4(a)-4(c)]. Calcium phosphate deposition was only weakly observed at seven days and slightly increased at 14 days, especially in micro-pores. Continuous culture also enhanced calcium deposition in macro-pores, indicating that AFSs permitted proliferation and differentiation using different sizes of pores.

Furthermore, to analyze chondrocyte hypertrophy in AFSs, we stained proteoglycans with Alcian Blue [Figs. 4(d)-4(f)], which showed that the cells were surrounded by a sulfated proteoglycan-rich ECM in micro-pores after reaching confluence at
14 days. Longer-term culture (at 21 days) enabled increased production of ECM in macro-pores at periphery [asterisks in Fig. 4(f)] of AFS that permitted calcium deposition. These results suggest that the AFS environment may be suitable for cell proliferation and differentiation into osteocytes.

\subsection{Relative expression levels of marker genes of osteogenic differentiation}

To investigate differential gene expression of BMSCs cultured in AFSs, semi-quantitative RT-PCR analyses of osteogenic differ- 
(a)

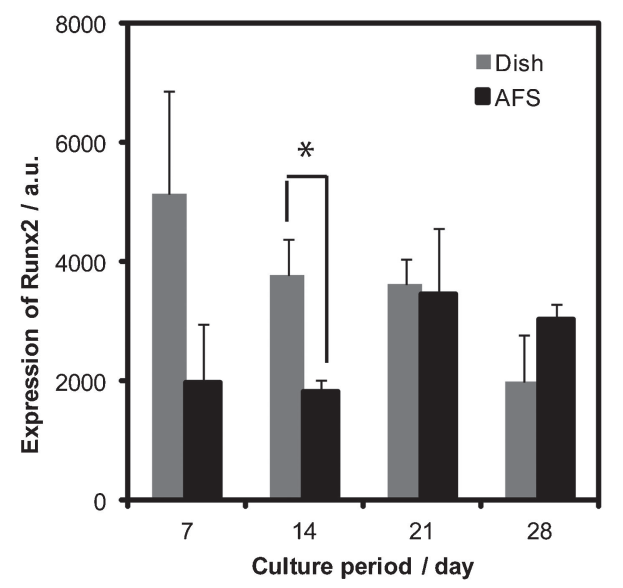

(c)

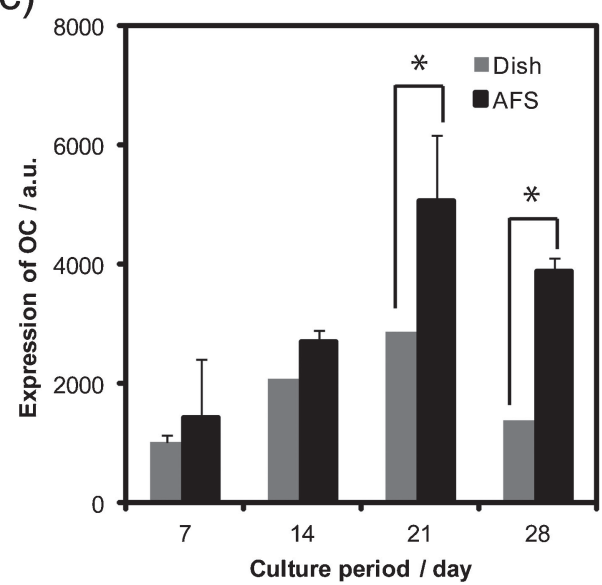

(b)

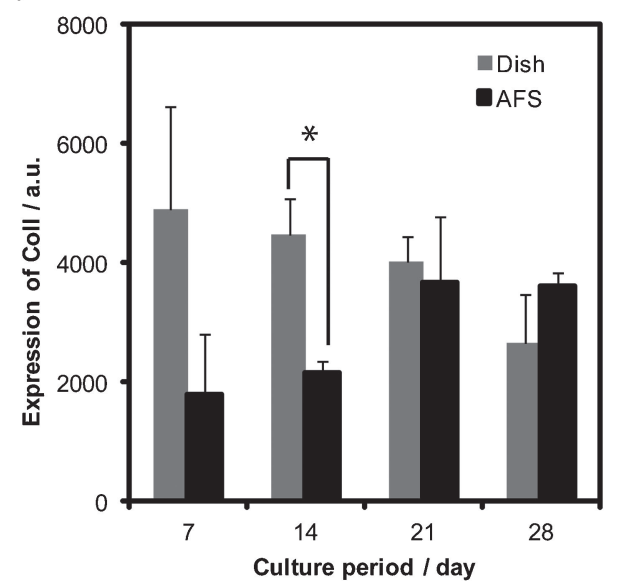

Fig. 5. Expression of osteogenic marker genes of BMSCs-seeded dish or AFS. BMSCs were cultured in dish or on AFS for $7,14,21$, or 28 days in normal growth medium. Total RNA was isolated and PCR was used to analyze for gene characteristics for the osteoblastic phenotype. The asterisks show ${ }^{*} P<0.05$ by Student's $t$-test.

entiation markers were carried out at defined time points $(7,14$, 21 , and 28 days).

Recent studies have shown that microstructures such as bonelike apatite layer formation and cell adhesion are essential for bone formation. ${ }^{21)}$ Elucidating the role microstructures play in bone differentiation is important. The expression of marker genes such as Runx2 and Col I in cells grown in the dish peaked at seven days and then decreased, indicating that two-dimensional homogenous cell proliferation promoted the progression of differentiation at an early time point [Figs. 5(a) and 5(b); gray bar]

In comparison, BMSCs in AFSs showed a different expression pattern. We observed two peaks in gene expression of Runx2 and Col I at both seven and 21 days in AFSs [Figs. 5(a) and 5(b); black bar]. BMSCs in macro-pores had differentiated at 21 days after proliferating, whereas cells in micro-pores began to differentiate after seven days. These findings showed that the first peak in gene expression was due to cell differentiation in micro-pores, and that the second peak was due to differentiation in macropores. On the other hand, the expression levels of $O C$ between cells grown in the dish and AFSs were significantly different [Fig. 5(c)], showing that the microstructures of the AFS strongly influenced calcification. These data are consistent with Alizarin Red staining [Figs. 4(a)-4(c)].

We conclude that osteogenic differentiation in AFSs was facilitated by the three-dimensional culture environment with two different sizes of pores and by the excellent biocompatibility of HAp.

\subsection{Relative expression levels of marker genes of endochondral ossification}

Bone development occurs via two distinct cellular and molecular pathways: intramembranous and endochondral ossification. ${ }^{22), 23)}$ The majority of in vivo bone formation occurs via endochondral ossification. To elucidate the pathway of bone differentiation in AFSs and to compare the gene expression profile between cells grown in the dish and the AFS, we analyzed the expression of endochondral ossification genes with semiquantitative RT-PCR at defined time points (7-28 days) in the regular medium (Fig. 6). Sox9 (early chondrogenic transcription factor) and aggrecan (marker of late-stage chondrocyte hypertrophy) were used as markers of chondrogenesis. Sox9 regulates Col II and aggrecan. ${ }^{24)}$

In the present study, the expression of $\operatorname{Sox} 9$ in cells grown in the dish peaked at seven days and then decreased. However, the levels of aggrecan mRNA in cells grown in the dish continued to increase up to 21 days. Aggrecan expression in cells grown in the dish was observed at a later time point compared with other marker genes. On the other hand, the expression of Sox9 on cells grown in AFSs also had two peaks, in agreement with expression of Runx2 and $\mathrm{Col} \mathrm{I}$. The expression level of aggrecan was increased and sustained during the culture period. 
(a)

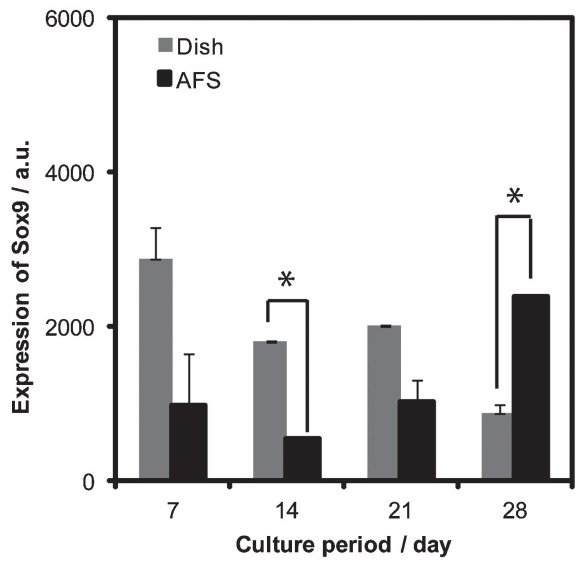

(c)

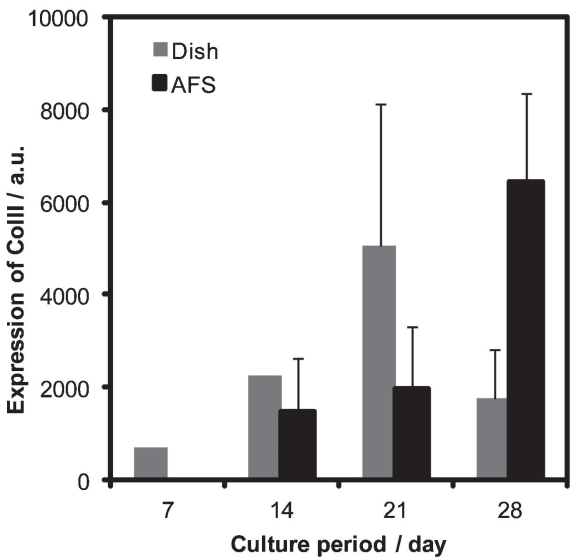

(b)

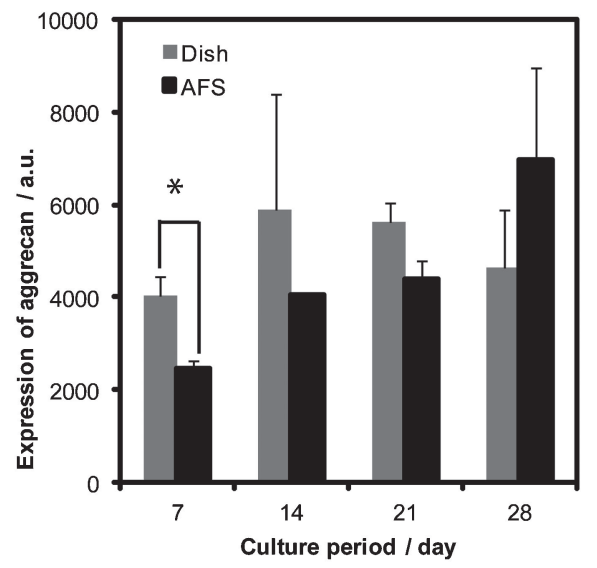

(d)

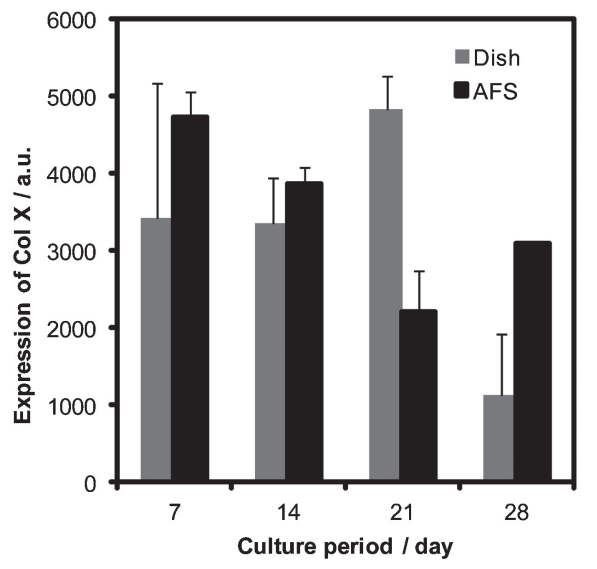

Fig. 6. Expression of endochondral ossification marker genes of BMSCs-seeded dish or AFS. BMSCs were cultured on dish or AFS for 7, 14, 21, or 28 days in normal growth medium. Total RNA was isolated and PCR was used to analyze for gene characteristics for the endochondral ossification phenotype. The asterisks show ${ }^{*} P<0.05$ by Student's $t$-test.

BMSCs cultured on AFSs in regular medium differentiated into a chondrogenic phenotype, as evidenced by chondrocyte-specific gene expression and by the synthesis of cartilage-associated ECM proteins.

On the other hand, the expression levels of Col II (marker of chondrocytes) and Col $X$ (marker of hypertrophic chondrocytes) were also enhanced in cells grown in AFSs compared with the levels in cells grown in dishes for a long culture period (28 days) [Figs. 6(c) and 6(d)]. In this study, the increase in Col X mRNA ceased after seven days, and the expression decreased afterwards. Col II continued to increase throughout the entire experimental period in cells grown in AFSs. These results suggest that BMSCs can differentiate into mature chondrocytes but not into hypertrophic chondrocytes at up to 28 days of culture in threedimensional AFSs. Therefore, bone differentiation on AFSs was achieved via endochondral ossification.

\subsection{Selective differentiation into osteocytes on AFSs}

MSCs isolated from a variety of adult tissues including bone marrow have the capacity to differentiate into different cell types, such as bone, cartilage, muscle, and fat, and have therefore attracted scientific interest as therapeutic tools for tissue repair. ${ }^{25)}$ Previous studies have shown that BMSCs are pluripotent progenitor cells that can regenerate different skeletal tissues in response to environmental signals. ${ }^{26), 27)}$ To investigate if BMSCs cultured in
AFSs differentiated toward multiple lineages, Sudan IV staining for adipocytes was carried out (Fig. 7). Numerous adipocyte foci were observed in culture dishes after 21 days [Figs. 7(b) and 7(d)]. However, BMSCs cultured in AFSs did not undergo adipogenesis. These data imply that AFSs selectively induced differentiation into osteocytes. The characteristic structure and chemical composition of HAp may contribute to enhancing the selectivity of BMSC differentiation into osteocytes.

\section{Conclusion}

Ideally, biomaterials for application in tissue engineering and regenerative medicine should both mimic the intended in vivo biochemical and biomechanical environment and support cell viability and phenotypic characterization. In the present study, we investigated differentiation of BMSCs in a three-dimensional scaffold and demonstrated that AFSs, which are composed of a three-dimensional porous matrix, would be potentially beneficial for bone regeneration.

Bone differentiation on AFSs occurred via endochondral ossification, as does the majority of in vivo bone formation. The characteristic microstructure of AFSs enhanced and sustained osteogenic differentiation. In addition, BMSCs cultured on AFSs were inhibited from differentiating into adipocytes, suggesting that AFSs selectively induced differentiation into osteoblasts. Increased understanding of the interaction between cells and scaffolds is fundamental for improving tissue engineering strategies. 

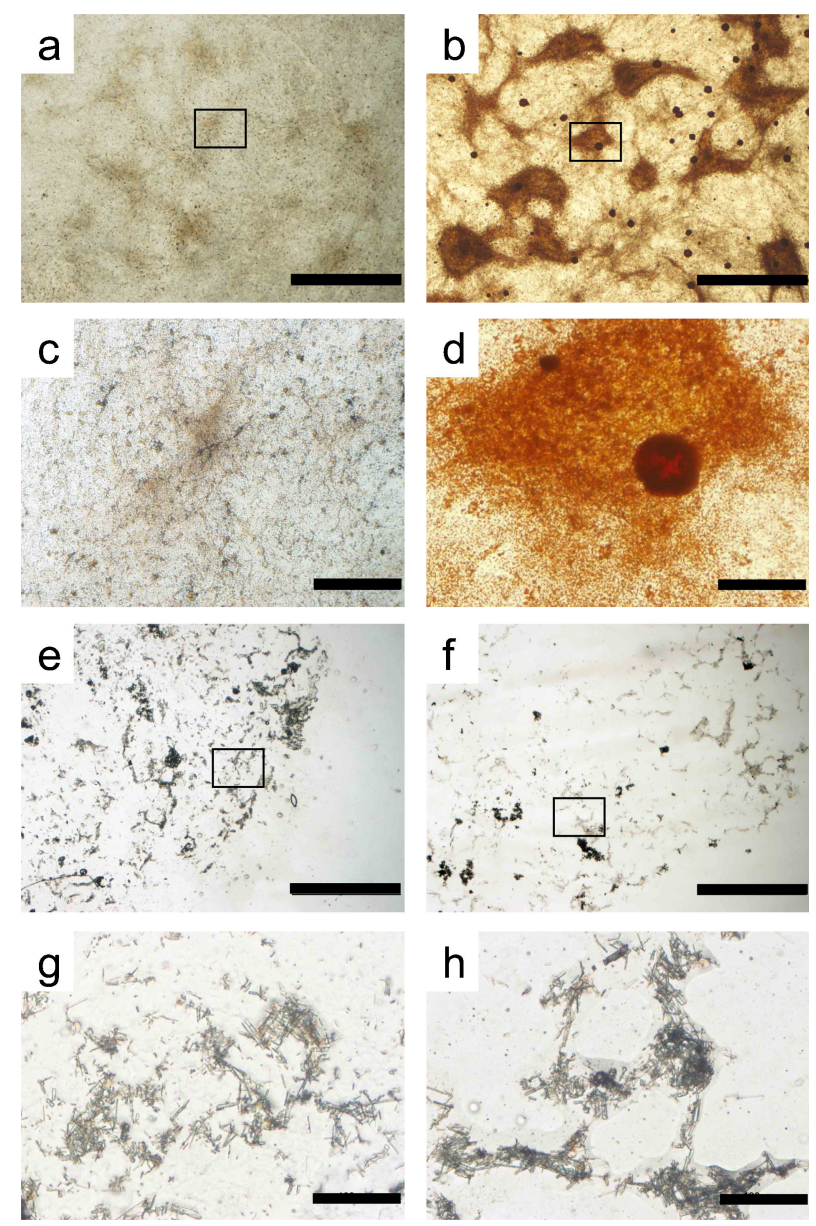

Fig. 7. Inhibition of adipogenic differentiation of BMSCs on AFS BMSCs were cultured in normal growth medium in culture dishes $(a-d)$ or on AFS (e-h) for 14 (a, c, e, g) or 21 (b, d, f, h) days. Thin sections were reacted with Sudan IV solution. Adipocytes were stained by orange color. Each area indicated by a square is magnified. Bars indicate $1 \mathrm{~mm}$ (a, b, e, f) or $100 \mu \mathrm{m}(\mathrm{c}, \mathrm{d}, \mathrm{g}, \mathrm{h})$.

\section{References}

1) H. Chim, J. T. Schantz and A. K. Gosain, Plast. Reconstr. Surg., 122, 755-764 (2008).

2) T. J. Heino and T. A. Hentunen, Curr. Stem Cell Res. Ther., 3, 131-145 (2008).

3) A. J. Salgado, O. P. Coutinho and R. L. Reis, Macromol. Biosci., 4, 743-765 (2004).

4) S. Srouji, T. Kizhner and E. Livne, Regen. Med., 1, 519-528 (2006).

5) M. Rumpler, A. Woesz, F. Varga, I. Manjubala, K. Klaushofer and P. Fratzl, J. Biomed. Mater. Res., Part A, 81, 40-50 (2007).

6) S. Srouji, T. Kizhner and E. Livne, Regen. Med., 1, 519-528 (2006).

7) A. D. Thompson, M. W. Betz, D. M. Yoon and J. P. Fisher, Tissue Eng. Part A, 15, 1181-1190 (2008).

8) M. Aizawa, A. E. Porter, S. M. Best and W. Bonfield, Biomaterials, 26, 3427-3433 (2005).

9) M. Aizawa, H. Ueno, K. Itatani and I. Okada, J. Eur. Ceram. Soc., 26, 501-507 (2006).

10) M. Aizawa, H. Shinoda, H. Uchida, I. Okada, T. J. Fujimi, N. Kanzawa, H. Morisue, M. Matsumoto and Y. Toyama, Phosphorus Res. Bull., 17, 262-268 (2004).

11) M. Honda, T. J. Fujimi, S. Izumi, K. Izawa, M. Aizawa, H. Morisue, T. Tsuchiya and N. Kanzawa, J. Biomed. Mater. Res., Part A, 94, 937-944 (2010).

12) D. J. Prockop, Science, 276, 71-74 (1997).

13) T. Hou, J. Xu, Q. Li, J. Feng and L. Zen, Tissue Eng. Part A, 14, 1173-1182 (2008).

14) R. Cancedda, M. Mastrogiacomo, G. Bianchi, A. Derubeis, A. Muraglia and R. Quarto, Novartis Found. Symp., 249, 133-143 (2003).

15) Y. Jiang, B. N. Jahagirdar, R. L. Reinhardt, R. E. Schwartz, C. D. Keene, X. R. Ortiz-Gonzalez, M. Reyes, T. Lenvik, T. Lund, M. Blackstad, J. Du, S. Aldrich, A. Lisberg, W. C. Low, D. A. Largaespada and C. M. Verfaillie, Nature, 418, 41-49 (2002).

16) G. Karsenty and E. F. Wagner, Dev. Cell, 2, 389-406 (2002).

17) H. M. Kronenberg, Nature, 423, 332-336 (2003).

18) S. Provot and E. Schipani, Biochem. Biophys. Res. Commun., 328, 658-665 (2005).

19) M. Aizawa, F. S. Howell, K. Itatani, Y. Yokogawa, K. Nishizawa, M. Toriyama and T. Kameyama, J. Ceram. Soc. Japan, 108, 249-253 (2000).

20) C. Maniatopoulos, J. Sodek and A. H. Melcher, Cell Tissue Res., 254, 317-330 (1988).

21) X. Li, C. A. van Blitterswijk, Q. Feng, F. Cui and F. Watari, Biomaterials, 29, 3306-3316 (2008).

22) K. Hoshi, T. Komori and H. Ozawa, Bone, 25, 639-651 (1999).

23) F. Rauch, R. Travers, A. M. Parfitt and F. H. Glorieux, Bone, 26, 581-589 (2000).

24) H. Tsuchiya, H. Kitoh, F. Sugiura and N. Ishiguro, Biochem. Biophys. Res. Commun., 301, 338-343 (2003).

25) C. Campagnoli, I. A. Roberts, S. Kumar, P. R. Bennett, I. Bellantuono and N. M. Fisk, Blood, 98, 2396-2402 (2001).

26) V. Nelea, L. Luo, C. N. Demers, J. Antoniou, A. Petit, S. Lerouge, R. Wertheimer and F. Mwale, J. Biomed. Mater. Res., Part A, 75, 216-223 (2005).

27) D. D. Deligianni, N. D. Katsala, P. G. Koutsoukos and Y. F. Missirlis, Biomaterials, 22, 87-96 (2001). 